\title{
Performance of Low-Dose Chest CT Scan for Initial Triage of COVID-19
}

\author{
Hooman Bahrami-Motlagh (iD) ${ }^{1,}$, Sahar Abbasi (iD ${ }^{1}$, Maryam Haghighimorad ${ }^{1}$, Babak Salevatipour (iD) ${ }^{1}$, \\ Ilad Alavi Darazam (D) ${ }^{2,3}$, Morteza Sanei Taheri (iD ${ }^{4}$, Farzad Esmaeili Tarki ${ }^{5}$ and Seyed Sina Naghibi \\ Irvani $^{1}$ \\ ${ }^{1}$ Department of Radiology, Loghman Hakim Hospital, Shahid Beheshti University of Medical Sciences, Tehran, Iran \\ ${ }^{2}$ Department of Infectious Diseases and Tropical Medicine, Loghman Hakim Hospital, Shahid Beheshti University of Medical Sciences, Tehran, Iran \\ ${ }^{3}$ Infectious Diseases and Tropical Medicine Research Center, Shahid Beheshti University of Medical Sciences, Tehran, Iran \\ ${ }^{4}$ Department of Radiology, Shohada Tajrish Hospital, Shahid Beheshti University of Medical Sciences, Tehran, Iran \\ ${ }^{5}$ Pediatric Gastroenterology, Hepatology and Nutrition Center, Research Institute for Children's Health, Shahid Beheshti University of Medical Sciences, Tehran, Iran \\ Corresponding author: Department of Radiology, Loghman Hakim Hospital, Shahid Beheshti University of Medical Sciences, Tehran, Iran. Email: bahrami@sbmu.ac.ir
}

Received 2020 May 13; Revised 2020 August 11; Accepted 2020 August 16.

\begin{abstract}
Background: Chest computed tomography (CT) scan is frequently used for diagnosis of coronavirus disease 2019 (COVID-19), especially in regions with limited availability of reverse-transcription polymerase chain reaction test (RT-PCR) test. Low-dose CT of chest offers acceptable image quality with lower radiation dose, particularly important in younger patients.

Objectives: We have designed the current study to evaluate the diagnostic efficacy of low-dose chest CT versus early RT-PCR results, for triage of COVID-19 patients.

Patients and Methods: From February 20 to April 15, 2020, 163 patients including 100 males (61.3\%) with the median age of 65 years (21 to 97), who underwent both RT-PCR and chest CT were registered in the study. Low-dose chest CT protocol was applied with parameters modified from the lung cancer screening protocol. The accuracy of low-dose chest CT for COVID-19 diagnosis was evaluated, considering first RT-PCR results as reference.

Results: Of 163 patients, 89 cases (54.6\%) were presented with positive initial RT-PCR result. Lymphocyte percentage and lymphocyte count were significantly lower in the positive RT-PCR group (15\% versus $19 \%$, and 0.98 vs. 1.3, respectively); while, erythrocyte sedimentation rate (ESR) was significantly higher (53 vs. 22). Positive chest CT findings were present in 133/163 cases (81.6\%). The sensitivity, specificity, positive and negative predictive values (PPV and NPV) and accuracy of low-dose chest CT scan were $96.6 \%$ (95\% confidence interval [CI], 90\% - 99\%), 36.5\% (95\% CI, 26\% - 49\%), 64.7\% (95\% CI, 56\% - 73\%), 90\% (95\% CI, 72\% - 97\%) and 69.3\% (95\% CI, 61\% - 76\%), respectively based on positive RT-PCR results.

Conclusion: Low-dose chest CT scan provides both high sensitivity and negative predictive value in diagnosing COVID-19 compared to initial RT-PCR as the gold standard. It can be used as an alternate to standard-dose CT scan in areas with high prevalence of COVID19 disease and limited availability of RT-PCR for early triage.
\end{abstract}

Keywords: Novel Coronavirus 2019, COVID-19, Chest CT Scan, Low-Dose Protocol

\section{Background}

In the days leading up to the end of 2019, new coronavirus was declared as the cause of coronavirus disease 2019 (COVID-19) responsible for the viral pneumonia outbreak in Wuhan, China (1). The Chinese official let the world know that a virus was spreading inside their communities, which then in the second week of February 2020, the international authorities responsible in such incidents, chose "severe acute respiratory syndrome coronavirus 2" (SARSCoV-2) as the name for it. Accordingly, World Health Organization (WHO) recognized the related illness as "coronavirus disease 2019" (2). Ensuingly, it was determined that the current predicament is needed to be proclaimed as a pandemic (3). Appertaining to the WHO report on 11 June 2020 , over 7.2 million individuals globally, have definite diagnosis of COVID-19 and the world's death toll from the disease has currently surpassed 413000 which has resulted in a mortality rate of approximate 5.7 percent (4).

In Iran, first cases were reported in February 20, 2020. The severity of the situation was aggravated so quickly, that by 10 th of March, Iran was amongst the five countries with the highest prevalence and death tolls because of COVID-19 (5) and by 11th June remained within the first ten countries (4). This condition had the Iranian National Health Service faced an increasing urgent demand for diagnosing in- 
fected cases, for the purpose of further isolation and medical care.

Based on the previous guidelines, COVID-19-inducedpneumonia diagnosis should be verified by reverse transcription polymerase chain reaction (RT-PCR) as benchmark (6). Given the restrictions occurring with collection of specimens and kit performance, the RT-PCR overall positive rate for samples collected by throat swabs has been described around 30\% - 60\% when initially presented (7). The preparation time for RT-PCR results to become available is variable and might be long in centers without in situ facility for analysis, which is also an important determining factor for clinical management. Apart from limitations of RT-PCR, with the uprising rate of suspected cases in an outbreak, the adequate number of kits would not be available as fast as required. Alternatively, computed tomography (CT) scan as a more accessible method, presents rapid results, and is available in many countries including Iran (8). Chest CT scan was suggested to be a satisfactory tool for detecting COVID-19 pneumonia based on reports from China, showing a sensitivity of up to $97 \%$ (9). A recent meta-analysis has demonstrated a pooled sensitivity of $94 \%$; however, the positive predictive value of CT scan was questioned outside China where there is a low prevalence of disease (10).

At the same time, the radiation risk of mass CT examination must be taken into consideration, since young patients under 40 years old will require testing. In addition, follow-up CT examination might be required to monitor the disease progression with a reported mean of $4 \pm 1 \mathrm{CT}$ exams (ranging from 3 to 6 ) within a short interim of $4 \pm 1$ days (ranging from 1 to 8 days) (11). Thus, the cumulative radiation burden associated with repetitive CT examination is of concern.

Low-dose CT scan is approved for screening lung cancer with adequate quality (12). Encouraging data from a recent article demonstrated no breaks in double-strand-DNA with low-dose chest CT, as against standard-dose (13), particularly suggesting that the low-dose protocol is safer. A recent study has demonstrated high accuracy of low-dose chest CT in detection of COVID-19 (14). However, they have performed repeat RT-PCR tests in two consequent days, which is not easily available in many centers in our country.

\section{Objectives}

By conducting this research, we aimed to measure the accuracy of low-dose chest CT compared to first initial RTPCR results, for detection of SARS-CoV-2.

\section{Patients and Methods}

\subsection{Patients and Data Sources}

The current cross-sectional study had been granted approval by the institutional review board (IRB), while the requisite to obtain patient consent has been waived. From February 20 to April 15, suspected COVID-19 patients, which had both chest CT scan and RT-PCR assay were enrolled in this single-center analysis.

The criteria for performing chest CT scan was based on the presence of one of the following symptoms according to our local hospital guideline: (1) Fever with a temperature exceeding $38^{\circ}$ Celsius; (2) oxygen saturation of less than 93\%; (3) respiratory rate higher than $30 / \mathrm{min}$.

COVID-19 infection was confirmed with positive RT-PCR test, carried out by administering LightMix, SarbecoV Egene RT-PCR Kits (Roche, Berlin, Germany). The results took a median time of 24 hours to be ready. Demographic variables (age, gender, and clinical symptoms), laboratory data (blood cell count, ESR, C-reactive protein, aspartate aminotransferase, alanine aminotransferase, blood urea nitrogen and creatinine) and initial RT-PCR test results were obtained from our hospital information system (HIS) providing electronic medical records of cases.

In patients with multiple chest CT scans, the initial scan was included in the study. Patients, in whom the interval between RT-PCR test and chest CT exceeded 7 days, were excluded from the study.

\subsection{Chest CT Protocols}

While the COVID-19 disease outbreak took place in Iran, our tertiary referral center, encountered daily rises in the number of chest CT scan requests. A remarkable number of cases were younger than 40 years old and according to the Iranian Radiology Society (ISR) consensus (8), we applied a low-dose chest CT protocol mainly adjusted from lung cancer screening CT protocol (15). Therefore, patients presenting in earlier days, underwent standard-dose CT scan; whereas, more recent cases underwent the low-dose protocol. Scanning parameters are presented in Table 1.

Images were obtained with one of two CT scanners (Activion 16, Toshiba, Japan and Somatom scope power 16, Siemens Healthineers, Germany). The CT scan results were instantly transferred to picture archiving and communication system (PACS). Preliminary reports where available within 2 hours.

\subsection{Radiation Dose Measurement}

The volume of CT dose index (CTDIvol) in mGy, along with the dose-length product (DLP) in mGy.cm were obtained from the automatically generated dose report, 


\begin{tabular}{|c|c|c|c|c|}
\hline \multirow{2}{*}{$\begin{array}{l}\text { Vendor } \\
\text { Protocol }\end{array}$} & \multicolumn{2}{|c|}{ Activion 16, Canon } & \multicolumn{2}{|c|}{ Somatom 16, Siemens } \\
\hline & Low-dose & Standard-dose & Low-dose & Standard-dose \\
\hline Scan type & Helical & Helical & Spiral & Spiral \\
\hline Rotation time, $s$ & 0.75 & 0.75 & 0.6 & 0.6 \\
\hline Detector configuration, $\mathrm{mm}$ & $16 \times 1.0$ & $16 \times 1.0$ & $16 \times 1.2$ & $16 \times 1.2$ \\
\hline $\mathbf{k V}$ & 120 & 120 & 110 & 130 \\
\hline $\mathbf{m A}$ /reference $\mathbf{m A}$ & 30 & $80-500$ & 20 & 110 \\
\hline Sure exposure/CARE dose & ON & ON & ON & ON \\
\hline
\end{tabular}

based on the specific manufacturing company. Effective dose in millisievert $(\mathrm{mSv})$ was computed by DLP $\times \mathrm{k}$ formula, with $\mathrm{k}$ (the fraction of $\mathrm{mSv}$ over $\mathrm{mGy} . \mathrm{cm}$ ) assigned as 0.014 for chest CT according to the 2008 report of American Association of Physicists in Medicine (16).

\subsection{Image Analysis}

Two radiologists (B.S. and M.H. with 12 and 10 years experience, respectively in reporting chest CT scan) who were blinded to RT-PCR results and clinical symptoms, analyzed all images independently. They reported negative or positive CT, according to previous reports on typical and atypical CT findings of COVID-19 pneumonia (17). If the intended accordance was absent, both radiologists would carry out the read-out in concord.

\subsection{Statistical Analysis}

The statistical assessment was accomplished by employing version 21.0 of SPSS (IBM Corp. Released 2012. IBM SPSS Statistics for Windows, Armonk, NY). To display parameters, categorical variables were detailed as percentages and counts, and continuous variables were shown as mean \pm standard deviation.

The accuracy, negative predictive value (NPV), positive predictive value (PPV), specificity, sensitivity, positive and negative likelihood ratio of low-dose chest CT were computed using RT-PCR outcomes as the reference. Comparison of quantitative data was performed using Student $\mathrm{T}$ test or Mann-Whitney U-test, according to the normal distribution assessed by Kolmogorov-SmirnovZ test. A P value of less than 0.05 was considered as statistical significance.

\section{Results}

\subsection{Demographic Characteristics and Laboratory Data}

Amongst 205 patients with both RT-PCR and CT scan results available, 42 patients had chest CT scan performed with standard-dose, acquired before the low-dose protocol was implemented or alternatively because COVID-19 pneumonia was not suspected at the initial presentation. A total of 163 patients with low-dose CT protocol were enrolled into the study (Figure 1) including 63 female (38.7\%) and 100 male (61.3\%) cases with the median age of 65 (ranging from 21 to 97) years. Among 163 patients, 28 (17.2\%) were 40 years old or younger. All patients had one RT-PCR assay and at least one CT scan, with 16 patients with two or more CT scans. The mean interval between commencing of symptoms and RT-PCR testing was $4 \pm 4$ days, whereas the mean interval between commencing of symptoms and CT was 2 \pm 2 days. Of 163 cases, 89 (54.6\%) had positive results of RTPCR for COVID-19. Within those 89 patients with confirmed diagnosis of COVID-19, more frequent clinical symptoms were dyspnea (75/89, 84.3\%), fever (68/89, 76.4\%), and cough (65/89, 73\%). Demographic and laboratory results are presented in Table 2.

\subsection{CT Scan Findings}

Of 163 patients, 151 (92.6\%) had abnormal findings in CT scan at baseline. Among these 151 cases with abnormal CT, 133 (81.6\%) were presented with typical CT findings of COVID-19 viral pneumonia assigned as positive. Most frequent findings included ground-glass opacity and consolidation mostly bilateral in peripheral/subpleural location (Table 3).

Out of 163 patients, 18 (11.0\%) had CT manifestations other than viral pneumonia including bacterial pneumonia, aspiration pneumonia (consolidation in dependent portion of the lung fields) or bronchiolitis (centrilobular nodular infiltration). Twelve patients (7.4\%) had normal CT scan.

Compared to RT-PCR as the standard reference, the sensitivity, specificity, PPV, NPV, and accuracy of low-dose chest CT scan were 96.6\% (95\% confidence interval [CI], 90\% 99\%), 36.5\% (95\% CI, 26\% - 49\%), 64.7\% (95\% CI, 56\% - 73\%), 90\% (95\% CI, 72\% - 97\%) and 69.3\% (95\% CI, 61\% - 76\%), respectively. Three cases with false negative CT results had normal CT scans. Positive and negative likelihood ratios were 1.52 


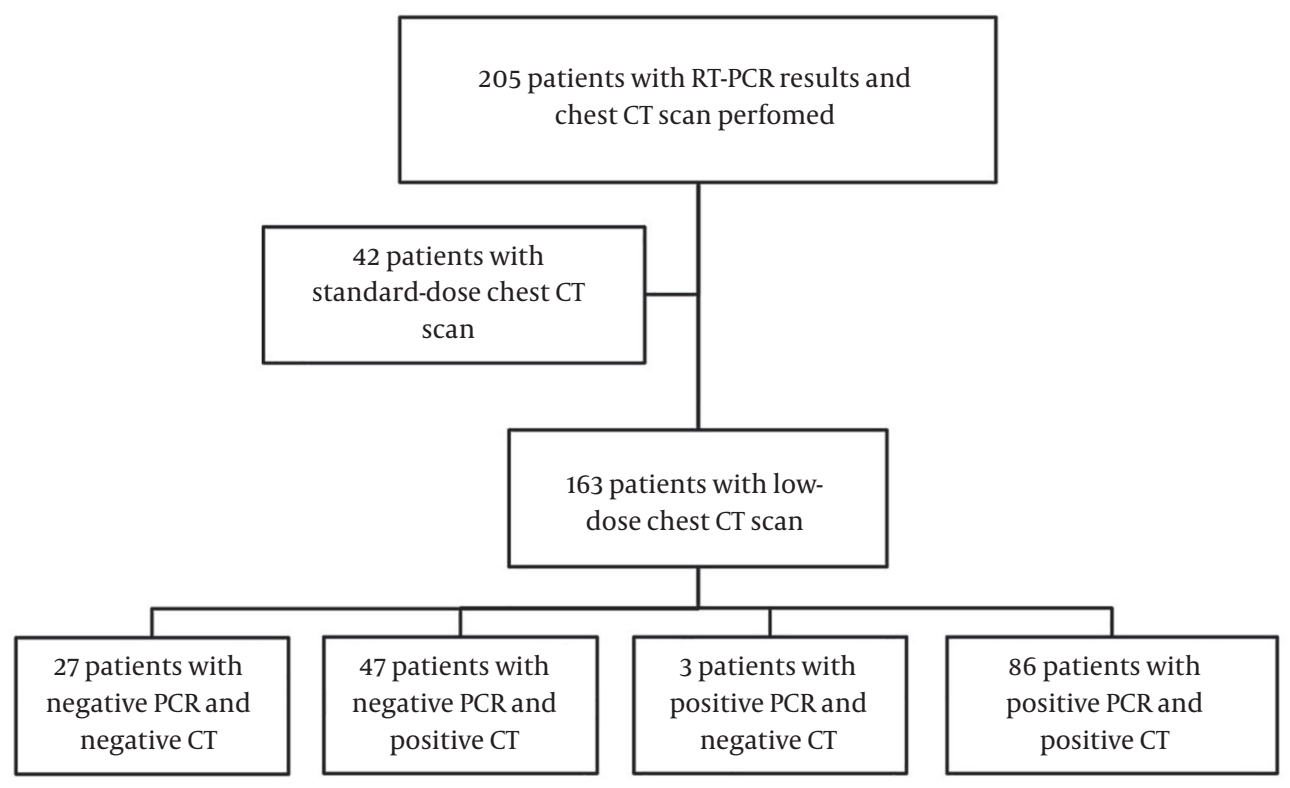

Figure 1. Flowchart of patient enrollment and number of patients in each subgroup considering PCR and CT scan results together

\begin{tabular}{|c|c|c|c|}
\hline & Positive PCR $(\mathrm{N}=\mathbf{8 9})$ & Negative PCR $(N=74)$ & P Value \\
\hline Age $^{\mathrm{b}}$ & $65 \pm 18$ & $57 \pm 18$ & 0.007 \\
\hline Gender, Male, \% & $53(59.6)$ & $47(63.5)$ & 0.631 \\
\hline \multicolumn{4}{|l|}{ Clinical symptoms } \\
\hline Dyspnea, \% & $75(84.3)$ & $53(71.6)$ & 0.057 \\
\hline Fever, \% & $68(76.4)$ & $44(59.5)$ & 0.027 \\
\hline Cough, $\%$ & $65(73)$ & $39(52.7)$ & 0.009 \\
\hline White blood cell count, G/L & $7.5 \pm 3.4$ & $7.7 \pm 4.6$ & 0.856 \\
\hline Lymphocyte count, G/L $\mathbf{L}^{\mathbf{b}}$ & $0.98 \pm 0.5$ & $1.3 \pm 0.6$ & 0.002 \\
\hline Lymphocyte percentage, $\%$ & $15 \pm 9$ & $19 \pm 10$ & 0.006 \\
\hline Erythrocyte sedimentation rate, $\mathbf{m m} / \mathbf{h}^{\mathrm{b}}$ & $53 \pm 36$ & $22 \pm 24$ & 0.001 \\
\hline C-reactive protein, $\mathrm{mg} / \mathrm{L}$ & $54 \pm 34$ & $56 \pm 39$ & 0.844 \\
\hline Aspartate aminotransferase, $\mathrm{U} / \mathrm{L}$ & $64 \pm 53$ & $72 \pm 82$ & 0.510 \\
\hline Alanine aminotransferase, $\mathrm{U} / \mathrm{L}$ & $55 \pm 36$ & $70 \pm 98$ & 0.979 \\
\hline Blood urea nitrogen, mg/dL & $61 \pm 54$ & $45 \pm 26$ & 0.146 \\
\hline Creatinine, $\mathrm{mg} / \mathrm{dL}$ & $1.5 \pm 1$ & $1.3 \pm 0.6$ & 0.515 \\
\hline
\end{tabular}

Abbreviation: PCR, polymerase chain reaction

${ }^{\mathrm{a}}$ Values are expressed as mean \pm SD or No. (\%).

${ }^{\mathrm{b}}$ Significant result with P value $<0.05$ 


\begin{tabular}{|lc|}
\hline \multicolumn{3}{l}{ Table 3. Chest CT Findings of 109 Patients with Low-Dose Protocol } \\
\hline \multicolumn{2}{|c|}{ Values } \\
\hline Consistent with viral pneumonia (positive) & $133 / 163(81.6 \%)$ \\
\hline Findings & \\
\hline & $122 / 133(91.7 \%)$ \\
\hline Ground glass opacity & $28 / 133(21.1 \%)$ \\
\hline Crazy paving & $80 / 133(60.2 \%)$ \\
\hline Nodular infiltration & $7 / 133(5.3 \%)$ \\
\hline Reverse Halo & $2 / 133(1.5 \%)$ \\
\hline Lymphadenopathy & $4 / 133(3 \%)$ \\
\hline Pleural effusion & $6 / 133(4.5 \%)$ \\
\hline Distribution & \\
\hline Peripheral/subpleural & $91 / 133(68.4 \%)$ \\
\hline Central/peribronchovascular & $5 / 133(3.8 \%)$ \\
\hline Both peripheral and central & $37 / 133(27.8 \%)$ \\
\hline Right lung & $3 / 133(2.3 \%)$ \\
\hline Left lung & $3 / 133(2.3 \%)$ \\
\hline Bilateral & $127 / 133(95.5 \%)$ \\
\hline Inconsistent with viral pneumonia or normal \\
(negative) & $30 / 163(18.4 \%)$ \\
\hline
\end{tabular}

(95\% CI,1.27-1.81) and 0.09 (95\% CI, $0.02-0.29$ ), respectively. Subgroup analysis was performed regarding patient's gender and age, which are summarized in Table 4 .

The mean CTDIvol, DLP and effective dose of low-dose protocol were $1.77 \pm 0.7 \mathrm{mGy}, 64.7 \pm 23.7 \mathrm{mGy} . \mathrm{cm}$, and 0.91 $\pm 0.33 \mathrm{mSv}$, respectively. Overall, the radiation dose was remarkably lower than the observed amounts in 42 cases with standard-dose chest CT scan protocol(Figure1), which were $12.8 \pm 4.0 \mathrm{mGy}, 413 \pm 148 \mathrm{mGy} . \mathrm{cm}, 5.8 \pm 2.1 \mathrm{mSv}$, respectively (P values $<0.001)$.

\section{Discussion}

Initial diagnosis of COVID-19 has been of essential significance in controlling the condition and providing medical care. Recent studies have demonstrated characteristic imaging findings in chest $\mathrm{CT}$ scan with promising accuracy in the detection of COVID-19 (17-19). Although the American College of Radiology proposes that chest CT must not be utilized for screening COVID-19 and it has to be reserved for hospitalized, symptomatic cases with specific clinical indications (20), in more epidemic areas including China, the local health officials have permitted the physicians to make the diagnosis based upon clinical data and chest CT findings (21) due to inadequate PCR kits in certain centers along with the considerable chance of false negative RTPCR results. Also, a recent guideline from Fleischner Society has proposed that in epidemic regions with limited PCR test availability, an initial CT scan is indicated in suspected COVID-19 patients with moderate to severe symptoms for fast decision making (22). Similarly, the Iranian Society of Radiology suggested a low-dose chest CT protocol to carry out screening, especially in settings where the COVID-19 RTPCR diagnostic kits are scarce (8).

Given RT-PCR outcomes as reference in 163 cases, we found a sensitivity and NPV of $96.6 \%$ and $90 \%$, respectively, much the same as figures demonstrated by Ai et al. (9). In the mentioned study, a negative predictive value of $83 \%$ was indicated together with a sensitivity of $97 \%$. Meanwhile, the average effective radiation dose was five to six times lower in this study compared to similar standarddose chest CT exam.

The low-dose chest CT protocol applied in this research has been modified from an existing lung cancer screening CT protocol, version 5.1, initially rendered by the American Association of Physicists in Medicine (AAPM). The CTDIvol average value in our study was $1.77 \mathrm{mGy}$, which is within the accepted limits for low-dose protocol in 16-row detector CT devices (15). The tube current-time product in our study was between 20 -30 mAs with adequate quality of images (Figure 2). As described previously by Zhu et al. (23), comparison between the image quality of chest CT exams acquired at $25 \mathrm{mAs}$ and those acquired at $115 \mathrm{mAs}$ did not delineate substantial difference. Additionally, a higher percentage of images with normal-quality were seen in lung window setting compared to images with normal-quality in mediastinal window setting, which means better preserved quality in evaluating lung parenchymal abnormalities. Similarly, in the current study, even small peripheral patches of ground glass opacity were identified (Figure 3 ).

We had 3/89 patients with normal CT scan and positive RT-PCR test. All three cases had CT scans acquired within 2 days from the symptom onset and this may be related to early imaging. As described previously by Bernheim et al. (24), from the cases who obtained CT scan within the first 48 hours after symptom onset, up to $56 \%$ had normal CT. Besides, Ling et al. (25) demonstrated that of 295 individuals with a confirmed diagnosis of COVID-19, 49 (17\%) had a negative initial chest CT scan, while $34(12 \%)$ remained negative after 3-14 days and mostly with few clinical symptoms.

In our study, $63.5 \%$ (47/74) of patients with negative PCR results had typical CT findings of COVID-19, which is similar to a previous study by Ai et al. (9) reported as $70 \%$. CT results of COVID-19 may be overlapped by findings in non-COVID pneumonia (26). Nonetheless, considering the rapid spread of COVID-19, what must be prioritized is to identify suspicious cases, so that early separation of affected individuals and their contacts, along with administration of proper clinical care could become possible. Also, 


\begin{tabular}{|c|c|c|c|c|c|c|c|}
\hline & Sensitivity, \% & Specificity, \% & PPV, \% & NPV, \% & Accuracy, \% & PLR & NLR \\
\hline Value & 96.6 & 36.5 & 64.7 & 90 & 69.3 & 1.52 & 0.09 \\
\hline 95\% CI & $90-99$ & $26-49$ & $56-73$ & $72-97$ & $61-76$ & $1.27-1.81$ & $0.02-0.29$ \\
\hline \multicolumn{8}{|l|}{ Subgroups (n) } \\
\hline Male $(n=100)$ & 98.1 & 34 & 62.6 & 94.1 & 68 & 1.49 & 0.06 \\
\hline Female $(n=63)$ & 94.4 & 40.7 & 68 & 84.6 & 71.4 & 1.59 & 0.14 \\
\hline Age $\geq 60(n=94)$ & 100 & 36.1 & 71.6 & 100 & 75.5 & 1.57 & 0.00 \\
\hline
\end{tabular}

Abbreviations: CI, confidence interval; n, number of cases; NLR, negative likelihood ratio; NPV, negative predictive value; PLR, positive likelihood ratio; PPV, positive predictive value.
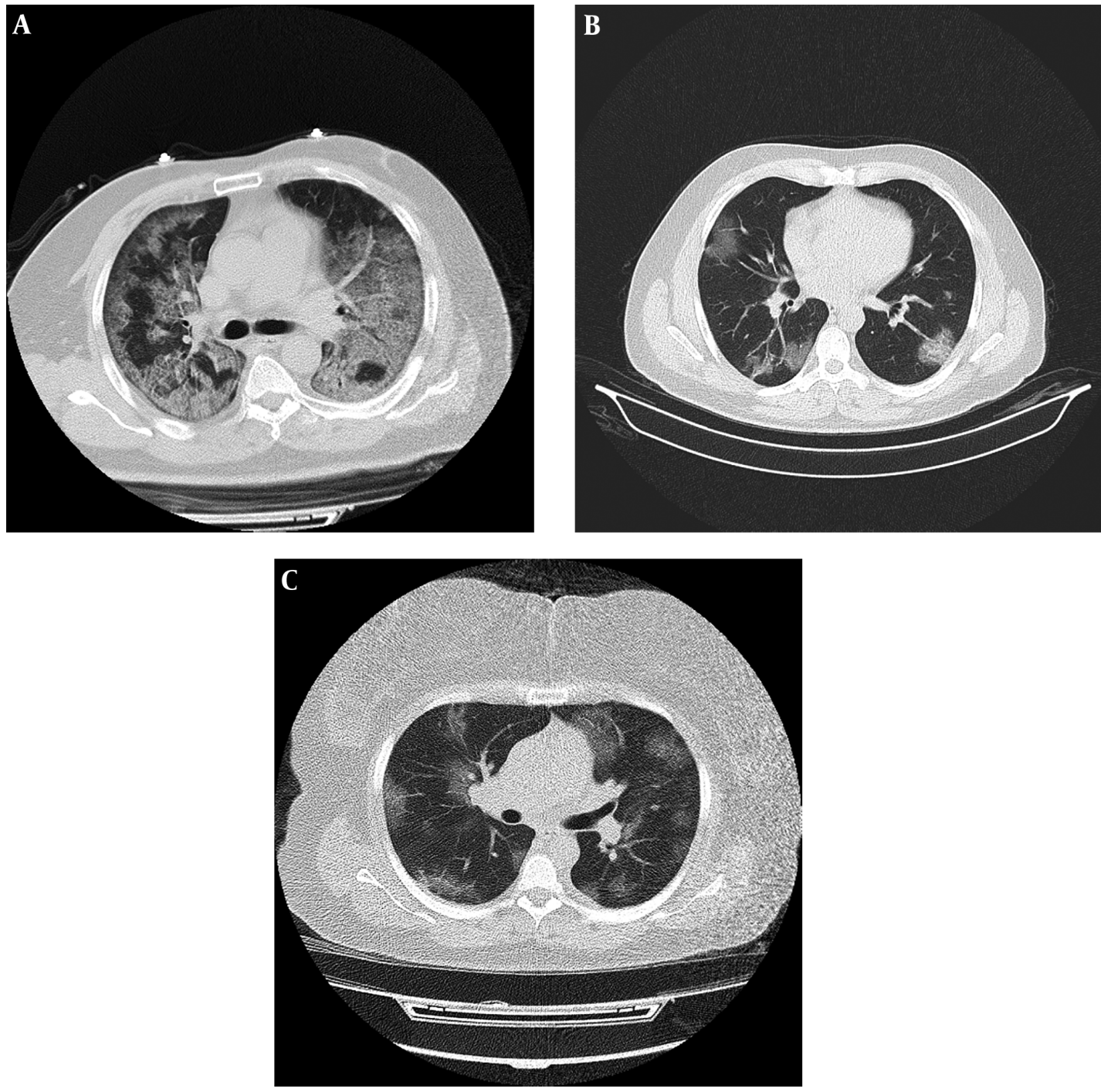

Figure 2. Examples of standard-dose and low-dose CT images with typical chest CT findings of COVID-19 pneumonia in patients with positive PCR. A, Fifty-seven years old male with fever and dyspnea for 10 days. Axial chest CT with standard-dose protocol (CTDIvol=15.9 mGy) shows bilateral subpleural and peribronchovascular ground glass opacities and crazy-paving; B, Thirty-six years old male with fever and cough for 2 days. Axial chest CT with low-dose protocol (CTDIvol =1.23) shows patchy bilateral peripheral ground glass opacities and early consolidation; C, Forty-eight years old female presenting with dyspnea for 4 days. Despite large body habitus of patient, axial chest CT with low-dose protocol (CTDIvol = 1.9) demonstrates multiple bilateral areas of peripheral and central ground glass opacities. 

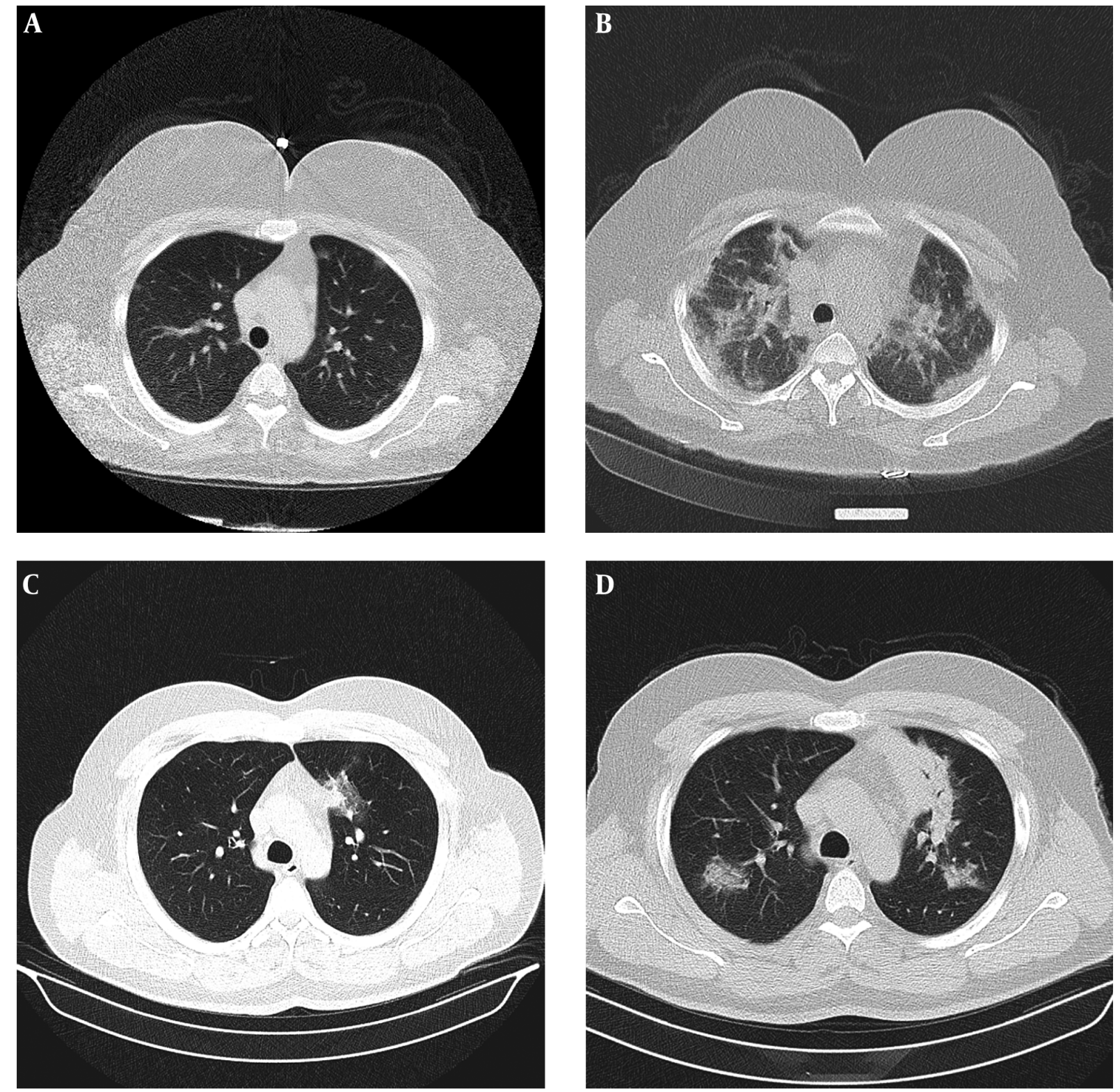

Figure 3. Progression of disease on serial studies. A, Axial chest CT with low-dose protocol (CTDIvol = $1.58 \mathrm{mGy}$ ) in 49 years old female with fever and dyspnea for 1 day shows small bilateral subpleural ground glass opacities; B, Axial chest CT 6 days later demonstrates progression of peripheral and central consolidation; C, Thirty-eight years old male with dyspnea for 6 days, axial chest CT with low-dose protocol (CTDIvol = $1.8 \mathrm{mGy}$ ) demonstrates single focus of ground glass opacity in paramediastinal portion of left upper lobe; D, Axial chest CT 4 days later shows increase in size and number of patches with more consolidate appearance.

it should be kept in mind that some of the false-positive patients on CT might in fact be true-positive, particularly when the RT-PCR's low positive rate is taken into consideration (Figure 4) Accordingly, Long et al. (27) demonstrated that $6 / 36$ patients with positive chest CT scan had a negative initial RT-PCR, which became positive after second and third repeats.

A recent study by Dangis et al. (14) demonstrated a specificity of 93.6\% for detection of COVID-19 with low-dose chest CT, markedly higher than results gathered in a recent meta-analysis by Kim et al. (10), which stated a pooled specificity of 35\% (range 25\% - 56\%) from the studies with repeated PCR. Dangis et al. (14) claimed that their high accuracy was due to the repeat of RT-PCR test in the two following days; however, considering that previous studies with repeated RT-PCR results have not reached this high level of 

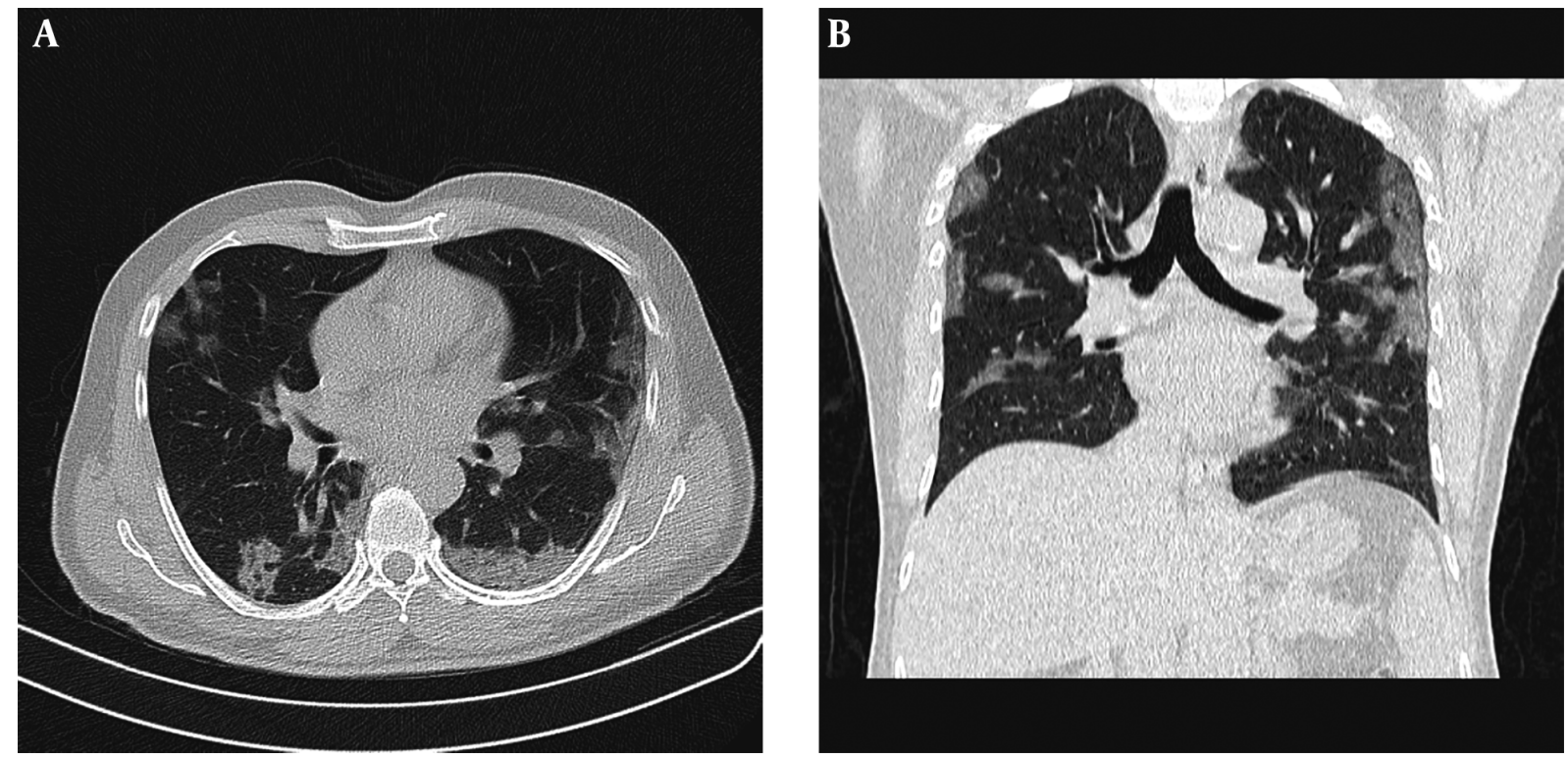

Figure 4. Example of positive chest CT scan findings for COVID-19 while negative PCR results. Fifty-four years old male with cough for 4 days, treated as COVID-19 according to clinical symptoms and CT findings. A, Axial; and B, Coronal chest CT with low-dose protocol (CTDIvol=1.01 mGy) shows bilateral subpleural and peribronchovascular ground glass opacities.

specificity, other possible explanations should be searched as well (28).

In the study by Dangis et al. (14), the accuracy of chest CT has not been compared to the first initial RT-PCR results, separately. In many hospital facilities in our country, there is relatively long delay for RT-PCR results to become available. We had a median waiting time of 24 hours in our center compared to few hours in the study by Dangis et al. (14). Hence, any repeat RT-PCR will double the time and become less practical from a clinical point of view.

Although it is important to finally prove COVID-19 infection with a positive PCR test, in our setting, with short supply to repeat RT-PCR, it was paramount for us to figure out the performance of low-dose CT compared to first initial PCR. We demonstrated the sensitivity and specificity of $96.6 \%$ and $36.5 \%$, respectively which are in concordance with the values for standard-dose chest CT gathered in a recent meta-analysis by Kim et al. (10) as $94 \%$ for sensitivity (95\% CI, 91\% - 96\%) and 37\% (95\% CI, 26\% - 50\%) for specificity.

Limitations in the current study include the following: First, we worked on a quite small quantity of cases. Second, in our study we could not directly compare low-dose CT accuracy with conventional CT, since there were only 42 patients who had both standard-dose CT and RT-PCR results in their profile. Therefore, we were not able to properly perform the non-inferiority test. Third, because of the shortage of RT-PCR kits, we could not repeat RT-PCR test, especially for negative cases, in the next following days. Therefore, we were not able to demonstrate that the results might eventually become positive somewhere down the process. Finally, we used RT-PCR tests as reference. Due to its rather small positive rate, the specificity of chest CT in SARS-CoV-2 cases might be understated; whereas, the sensitivity is overstated. Despite that, in an epidemic region, positive CT features, even with negative PCR results could still play a key clinical role in the rapid isolation of suspected cases for a better control of the viral spread. We believe that it is much needed to conduct further studies on this matter, due to its considerable clinical implication particularly in the low-resource settings where RT-PCR tests are not abundantly available for initial evaluation or to be repeated.

In conclusion, we have demonstrated that low-dose chest CT scan provides a high sensitivity and NPV in detecting COVID-19 pneumonia when compared to initial RT-PCR as the gold standard. Therefore, it might be considered as an alternate to standard-dose CT scan in epidemic areas with low availability of RT-PCR test.

\section{Acknowledgments}

The authors would like to thank Clinical Research Development Unit (CRDU) of Loghman Hakim Hospital, 
Shahid Beheshti University of Medical Sciences, Tehran, Iran for all the help and assistance.

\section{Footnotes}

Authors' Contributions: Conceptualization, methodology, formal analysis, writing- reviewing, and editing: HBM; investigation and original draft preparation: SA; data curation and resources: $\mathrm{MH}$; data curation, and supervision: BS; methodology, resources, writing-reviewing, and editing: IAD; validation, writing-reviewing, and editing: MST; writing-reviewing and editing: FET; resources, writing- reviewing, and editing: SSNI.

Conflict of Interests: The authors declare that they have no conflicts of interest regarding this study.

Ethical Approval: The current study had been granted approval by Institutional Review Board (IRB). The ethical approval code was IR.SBMU.RETECH.REC.1399.023.

Funding/Support: None declared.

Informed Consent: The requisite to obtain patient consent has been waived due to retrospective method of the study.

\section{References}

1. Huang C, Wang Y, Li X, Ren L, Zhao J, Hu Y, et al. Clinical features of patients infected with 2019 novel coronavirus in Wuhan, China. Lancet. 2020;395(10223):497-506. doi: 10.1016/s0140-6736(20)30183-5.

2. Rothan HA, Byrareddy SN. The epidemiology and pathogenesis of coronavirus disease (COVID-19) outbreak. J Autoimmun. 2020;109:102433. doi: 10.1016/j.jaut.2020.102433. [PubMed: 32113704]. [PubMed Central: PMC7127067].

3. Watkins J. Preventing a covid-19 pandemic. BMJ. 2020;368:m810. doi: 10.1136/bmj.m810. [PubMed: 32111649].

4. WHO. Coronavirus disease (COVID-19) Situation Report- 143. 2020, [updated 11 June 2020]. Available from: https://www.who.int/docs/ default-source/coronaviruse/situation-reports/20200611-covid19-sitrep-143.pdf?sfvrsn=2adbe568_6.

5. WHO. Coronavirus disease 2019 (COVID-19) Situation Report - 52. 2020, [updated 12 March 2020]. Available from: https://apps.who.int/iris/ handle/10665/331476.

6. CDC. Interim guidelines for collecting, handling, and testing clinical specimens from patients under investigation (PUIs) for Middle East respiratory syndrome coronavirus (MERS-CoV)-Version 2.1. 2015, [updated January 9, 2015]. Available from: https://prd-medweb-cdn. s3.amazonaws.com/documents/infectioncontrol/files/guidelinesclinical-specimens.pdf.

7. Yang Y, Yang M, Shen C, Wang F, Yuan J, Li J, et al. Evaluating the accuracy of different respiratory specimens in thelaboratory diagnosis and monitoring the viral shedding of 2019-nCoV infections. MedRxiv. 2020. doi: 10.1101/2020.02.11.20021493.

8. Mahdavi A, Khalili N, Davarpanah AH, Faghihi T, Mahdavi A, Haseli S, et al. Radiologic Management of COVID-19: Preliminary Experience of the Iranian Society of Radiology COVID-19 Consultant Group (ISRCC). Iran J Radiol. 2020;17(2). doi: 10.5812/iranjradiol.102324.

9. Ai T, Yang Z, Hou H, Zhan C, Chen C, Lv W, et al. Correlation of Chest CT and RT-PCR Testing for Coronavirus Disease 2019 (COVID19) in China: A Report of 1014 Cases. Radiology. 2020;296(2):E32-40. doi: 10.1148/radiol.2020200642. [PubMed: 32101510]. [PubMed Central: PMC7233399].

10. Kim H, Hong H, Yoon SH. Diagnostic Performance of CT and Reverse Transcriptase Polymerase Chain Reaction for Coronavirus Disease 2019: A Meta-Analysis. Radiology. 2020;296(3):E145-55. doi: 10.1148/radiol.2020201343. [PubMed: 32301646]. [PubMed Central: PMC7233409].

11. Pan F, Ye T, Sun P, Gui S, Liang B, Li L, et al. Time Course of Lung Changes at Chest CT during Recovery from Coronavirus Disease 2019 (COVID19). Radiology. 2020;295(3):715-21. doi: 10.1148/radiol.2020200370. [PubMed: 32053470]. [PubMed Central: PMC7233367].

12. Aberle DR, Adams AM, Berg CD, Black WC, Clapp JD; National Lung Screening Trial Research Team, et al. Reduced lung-cancer mortality with low-dose computed tomographic screening. $N$ Engl J Med. 2011;365(5):395-409. doi: 10.1056/NEJMoa1102873. [PubMed: 21714641]. [PubMed Central: PMC4356534].

13. Sakane H, Ishida M, Shi L, Fukumoto W, Sakai C, Miyata Y, et al. Biological Effects of Low-Dose Chest CT on Chromosomal DNA. Radiology. 2020;295(2):439-45. doi: 10.1148/radiol.2020190389. [PubMed: 32154776].

14. Dangis A, Gieraerts C, Bruecker YD, Janssen L, Valgaeren H, Obbels D, et al. Accuracy and reproducibility of low-dose submillisievert chest CT for the diagnosis of COVID-19. Radiol Cardiothorac Imaging. 2020;2(2). e200196. doi: $10.1148 /$ ryct.2020200196.

15. AAPM. Lung cancer screening CT protocols version 5.1. AAPM's working group on standardization of CT nomenclature and protocols. 2019, [updated 13 September 2019]. Available from: https://www.aapm.org/ pubs/CTProtocols/documents/LungCancerScreeningCT.pdf.

16. McCollough C, Cody D, Edyvean S, Geise R, Gould B, Keat N, et al. Report No. 096 - The Measurement, Reporting, and Management of Radiation Dose in CT(2008).AAPM; 2008. Available from: https://www.aapm. org/pubs/reports/detail.asp?docid=97.

17. Chung M, Bernheim A, Mei X, Zhang N, Huang M, Zeng X, et al. CT Imaging Features of 2019 Novel Coronavirus (2019-nCoV). Radiology. 2020;295(1):202-7. doi: 10.1148/radiol.2020200230. [PubMed: 32017661]. [PubMed Central: PMC7194022].

18. Lei J, Li J, Li X, Qi X. CT Imaging of the 2019 Novel Coronavirus (2019-nCoV) Pneumonia. Radiology. 2020;295(1):18. doi: 10.1148/radiol.2020200236. [PubMed: 32003646]. [PubMed Central: PMC7194019].

19. Shi H, Han X, Zheng C. Evolution of CT Manifestations in a Patient Recovered from 2019 Novel Coronavirus (2019-nCoV) Pneumonia in Wuhan, China. Radiology. 2020;295(1):20. doi: 10.1148/radiol.2020200269. [PubMed: 32032497]. [PubMed Central: PMC7233359].

20. ACo Radiology. ACR Recommendations for the use of Chest Radiography and Computed Tomography (CT) for Suspected COVID-19 Infection. ACR; 2020, [updated MARCH 22, 2020; cited March 11, 2020]. Available from: https://www.acr.org/Advocacy-and-Economics/ACRPosition-Statements/Recommendations-for-Chest-Radiographyand-CT-for-Suspected-COVID19-Infection.

21. Xinhua News Agency. China's Hubei reports jump in new cases of COVID-19 after diagnosis criteria revision. 2020, [cited 2020-02-13]. Available from: http://www.xinhuanet.com/english/2020-02/13/c_ 138779875.htm.

22. Rubin GD, Ryerson CJ, Haramati LB, Sverzellati N, Kanne JP, Raoof $\mathrm{S}$, et al. The Role of Chest Imaging in Patient Management during the COVID-19 Pandemic: A Multinational Consensus Statement from the Fleischner Society. Radiology. 2020;296(1):172-80. doi: 10.1148/radiol.2020201365. [PubMed: 32255413]. [PubMed Central: PMC7233395].

23. Zhu X, Yu J, Huang Z. Low-dose chest CT: optimizing radiation protection for patients. AJR Am J Roentgenol. 2004;183(3):809-16. doi: 10.2214/ajr.183.3.1830809. [PubMed: 15333374]. 
24. Bernheim A, Mei X, Huang M, Yang Y, Fayad ZA, Zhang N, et al. Chest CT Findings in Coronavirus Disease-19 (COVID-19): Relationship to Duration of Infection. Radiology. 2020;295(3):200463. doi: 10.1148/radiol.2020200463. [PubMed: 32077789]. [PubMed Central: PMC7233369].

25. Ling Z, Xu X, Gan Q, Zhang L, Luo L, Tang X, et al. Asymptomatic SARS-CoV-2 infected patients with persistent negative CT findings. Eur J Radiol. 2020;126:108956. doi: 10.1016/j.ejrad.2020.108956. [PubMed: 32199142]. [PubMed Central: PMC7118594 of interest].

26. Bai HX, Hsieh B, Xiong Z, Halsey K, Choi JW, Tran TML, et al. Performance of Radiologists in Differentiating COVID-19 from Non-
COVID-19 Viral Pneumonia at Chest CT. Radiology. 2020;296(2):E4654. doi: 10.1148/radiol.2020200823. [PubMed: 32155105]. [PubMed Central: PMC7233414].

27. Long $\mathrm{C}, \mathrm{Xu} \mathrm{H}$, Shen Q, Zhang X, Fan B, Wang C, et al. Diagnosis of the Coronavirus disease (COVID-19): rRT-PCR or CT? Eur J Radiol. 2020;126:108961. doi: 10.1016/j.ejrad.2020.108961. [PubMed: 32229322]. [PubMed Central: PMC7102545].

28. Bahrami-Motlagh $\mathrm{H}$, Sanei Taheri M, Abbasi S, Haghighimorad M, Salevatipour B, Alavi Darazam I. Accuracy of Low-Dose Chest CT Scan in Detection of COVID-19. Radiol Cardiothorac Imaging. 2020;2(3). e200256. doi: 10.1148/ryct.2020200256. 